\title{
Estimação de funções de covariância para características de crescimento da raça Tabapuã utilizando modelos de regressão aleatória
}

\section{Severino Cavalcante de Sousa Júnior ${ }^{1}$, Sônia Maria Pinheiro de Oliveira ${ }^{2}$, Lucia Galvão de Albuquerque $^{3,4}$, Arione Augusti Boligon ${ }^{5}$, Raimundo Martins Filho ${ }^{6}$}

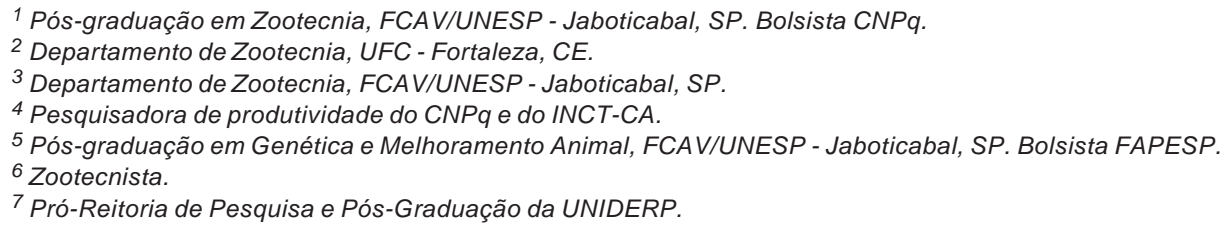

RESUMO - Foram utilizados 35.732 registros de peso do nascimento aos 660 dias de idade de 8.458 animais da raça Tabapuã para estimar funções de covariância utilizando modelos de regressão aleatória sobre polinômios de Legendre. Os modelos incluíram: como aleatórios, os efeitos genético aditivo direto, materno, de ambiente permanente de animal e materno; como fixos, os efeitos de grupo de contemporâneo; como covariáveis, a idade do animal à pesagem e a idade da vaca ao parto (linear e quadrática); e sobre a idade à pesagem, polinômio ortogonal de Legendre (regressão cúbica) foi considerado para modelar a curva média da população. O resíduo foi modelado considerando sete classes de variância e os modelos foram comparados pelos critérios de informação Bayesiano de Schwarz e Akaike. O melhor modelo apresentou ordens 4, 3, 6, 3 para os efeitos genético aditivo direto e materno, de ambiente permanente de animal e materno, respectivamente. As estimativas de covariância e herdabilidades, obtidas utilizando modelo bicaracter, e de regressão aleatória foram semelhantes. As estimativas de herdabilidade para o efeito genético aditivo direto, obtidas com o modelo de regressão aleatória, aumentaram do nascimento $(0,15)$ aos 660 dias de idade $(0,45)$. Maiores estimativas de herdabilidade materna foram obtidas para pesos medidos logo após o nascimento. As correlações genéticas variaram de moderadas a altas e diminuíram com o aumento da distância entre as pesagens. A seleção para maiores pesos em qualquer idade promove maior ganho de peso do nascimento aos 660 dias de idade.

Palavras-chave: correlação genética, dados longitudinais, gado de corte, herdabilidade, peso

\section{Estimation of covariance functions for growth traits in Tabapuã cattle using random regression models}

\begin{abstract}
In order to estimate covariance functions by using random regression models on Legendre polynomials, 35,732 weight records from birth to 660 days of age of 8,458 animals of Tabapuã cattle were used. The models included: as random effects, direct additive genetic effect, maternal effect, and animal and maternal permanent environmental effets; contemporary groups were included as fixed effects; and as covariables, animal age at weighing and age of dam at calving (linear and quadratic effects) and on age at weighing,, the orthogonal Legendre polynomial (cubic regression) was considered to model the mean curve of the population. Residuals effects were modeled considering seven classes of variance. The models were compared by Akaike criterion and Bayesian information criterion. The best model showed orders 4, 3, 6, and 3 for maternal and direct additive genetic effect, and maternal and animal permanent enviroment, respectively. Estimates of (co)variance and heritability obtained with the bi-trait and random regression models were similar. The heritability estimates for direct additive genetic effect obtained by the random regression models increased from birth (0.15) to 660 days of age (0.45). The greatest estimates of maternal heritability were obtained for weights measured right after birth. In general, the genetic correlation estimates were moderate to high, and they decreased as the distance between weights increased. Selection for higher weights at any age will promote weight gain from birth to 660 days of age.
\end{abstract}

Key Words: beef cattle, genetic correlations, heritability, longitudinal data, weights

Recebido em 25/11/2008 e aprovado em 7/5/2009. 


\section{Introdução}

O rebanho brasileiro da raça Tabapuã conta com um efetivo de aproximadamente 140.454 animais. O número de registros da raça tem aumentado nos últimos anos pelo fato destes animais apresentarem, além do caráter mocho, uma conformação do tipo cárneo sustentada por ossatura leve e robusta, resultando na produção de excelentes carcaças (ABCZ, 2008). A magnitude desses números confere a esta raça importância significativa na cadeia produtiva da carne brasileira e destaca a importância de estudos para avaliação do desempenho em nosso meio.

A avaliação genética de bovinos de corte depende, essencialmente, da disponibilidade das estimativas de parâmetros genéticos para as características de interesse econômico. Segundo Albuquerque \& El Faro (2008), os valores genéticos para características de crescimento em gado de corte são preditos e os componentes de variância são estimados considerando o peso às idades-padrão como, por exemplo, ao nascimento, à desmama, ao ano e ao final utilizando-se análises uni ou multicaracterísticas.

Nos últimos anos, os modelos de regressão aleatória estão sendo utilizados como alternativas às análises convencionais para estimar os componentes de variância e parâmetros genéticos de pesos de bovinos de corte obtidos em diferentes idades (Albuquerque \& Meyer, 2001; Nobre et al., 2003; Sakaguti et al., 2003; Dias et al., 2006).

Os modelos de regressão aleatória são casos especiais de funções de covariância que possibilitam estimar, de maneira direta, os coeficientes das funções de covariância pelo método de máxima verossimilhança restrita (Meyer \& Hill, 1997). Pelo estudo de dados longitudinais, como característica de crescimento, o uso de modelos de regressão aleatória permite a obtenção de parâmetros genéticos em qualquer idade dentro do intervalo considerado.

A utilização de modelos de regressão aleatória em características de pesos obtidos em diferentes idades possibilita a obtenção de DEP (diferenças esperadas na progênie) para qualquer idade do animal. Além disso, a utilização de modelos de regressão aleatória permite que a acurácia da avaliação seja aumentada, devido principalmente à eliminação de pré-ajustes nos dados e à possibilidade de se trabalhar com todas as pesagens disponíveis e com covariâncias adequadas.

O objetivo neste trabalho foi estimar parâmetros genéticos para pesos do nascimento aos 660 dias de idade de bovinos da raça Tabapuã oriundos do estado da Bahia, empregando modelos bicaracteres e de regressão aleatória.

\section{Material e Métodos}

Foram utilizadas 35.732 medidas de peso do nascimento aos 660 dias de idade, de 8.458 animais da raça Tabapuã criados no estado da Bahia, nascidos entre 1975 e 2001 e, provenientes da Associação Brasileira de Criadores de Zebu - ABCZ. Foram descartadas observações de animais com menos de três mensurações de peso. A média foi de seis pesagens por animal e o máximo oito. O grupo de contemporâneo foi definido por: sexo, mês e ano de nascimento, mês e ano da pesagem, fazenda e condição de criação. Foram consideradas duas condições de criação, ou seja, animais mamando sem ordenha e desmamados. Aplicou-se a restrição de que cada grupo de contemporâneo deveria conter, no mínimo, três animais, totalizando 2.615 grupos de contemporâneos.

Para melhor distribuição dos animais em cada idade, foram criadas classes com intervalos de cinco dias de idade em cada classe até 550 dias de idade, intervalo de dez dias de idade dos 561 aos 600 dias e de 30 dias dos 601 aos 660 dias, totalizando 118 classes de idades (Tabela 1).

Nos modelos, foram considerados fixos os efeitos de grupo de contemporâneo e, como covariáveis, a idade do animal à pesagem e idade da vaca ao parto (efeitos linear e quadrático). Como aleatórios, foram considerados os efeitos genéticos aditivo direto e materno, de ambiente permanente de animal e materno. Além disso, polinômio ortogonal de Legendre, de ordem cúbica, sobre a idade à pesagem foi considerado no modelo como efeito fixo, para modelar a curva média da população.

O modelo geral pode ser representado por:

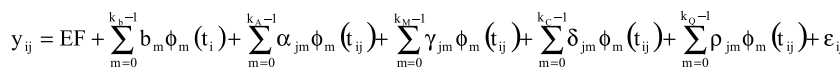
em que: $y_{i j}=i^{\text {śsima }}$ medida referente ao jésimo animal; $E F=$ conjunto de efeitos fixos; $b_{m}=$ coeficiente de regressão fixo para modelar a curva média da população; $\phi_{m}\left(t_{i}\right)=$ função

Tabela 1 - Estrutura do arquivo de dados

\begin{tabular}{lc}
\hline Análise dos dados & Número \\
\hline Total de registros & 35.732 \\
Animais na matriz de parentesco & 13.114 \\
Animais com registros & 8.458 \\
Animais com 3 observações & 3.678 \\
Animais com 4 observações & 5.152 \\
Animais com 5 observações & 4.906 \\
Animais com 6 observações & 8.824 \\
Animais com 7 observações & 10.978 \\
Animais com 8 observações & 4.197 \\
Touros & 167 \\
Vacas & 3.707 \\
Grupos de contemporâneos & 2.615 \\
\hline
\end{tabular}

R. Bras. Zootec., v.39, n.5, p.1037-1045, 2010 
de regressão que descreve a curva média da população de acordo com a idade do animal; $\phi_{m}\left(t_{i j}\right)=$ funções de regressão que descrevem as trajetórias de cada indivíduo j, de acordo com a idade $\left(\mathrm{t}_{\mathrm{i}}\right)$, para os efeitos aleatórios genético aditivo direto, aditivo materno, ambiente permanente do animal e ambiente permanente materno; $\alpha_{j m}, \gamma_{j m}, \delta_{j m}, \rho_{j m}=$ coeficientes de regressão genético aditivo direto e materno, de ambiente permanente de animal e materno, respectivamente, para cada animal; $\mathrm{k}_{\mathrm{b}}, \mathrm{k}_{\mathrm{A}}, \mathrm{k}_{\mathrm{M}}, \mathrm{k}_{\mathrm{C}}$ e $\mathrm{k}_{\mathrm{Q}}=$ ordem dos polinômios a serem ajustados; $\mathrm{e}_{\mathrm{ij}}=$ erro aleatório associado a cada idade $\mathrm{i}$ do animal $\mathrm{j}$.

Em notação matricial, o modelo utilizado pode ser descrito como:

$$
\begin{gathered}
\mathrm{Y}=\mathrm{X} \beta+\mathrm{Z}_{1} \alpha+\mathrm{Z}_{2} \gamma+\mathrm{W}_{1} \delta+\mathrm{W}_{2} \lambda+\varepsilon \\
\mathrm{V}\left[\begin{array}{l}
\alpha \\
\gamma \\
\delta \\
\lambda \\
\varepsilon
\end{array}\right]=\left[\begin{array}{ccccc}
\mathrm{K}_{A} \otimes A & 0 & 0 & 0 & 0 \\
0 & \mathrm{~K}_{M} \otimes A & 0 & 0 & 0 \\
0 & 0 & \mathrm{~K}_{C} \otimes \mathrm{I}_{N_{a}} & 0 & 0 \\
0 & 0 & 0 & \mathrm{~K}_{Q} \otimes \mathrm{I}_{N_{m}} & 0 \\
0 & 0 & 0 & 0 & R
\end{array}\right] ;
\end{gathered}
$$

em que: $Y=$ vetor de observações; $\beta=$ vetor de efeitos fixos; $\alpha=$ vetor aleatório dos coeficientes genéticos aditivos diretos; $\gamma=$ vetor aleatório dos coeficientes genéticos aditivos maternos; $\mathrm{d}=$ vetor de coeficientes de ambiente permanente de animal; $\lambda=$ vetor de coeficiente de ambiente permanente materno; $\mathrm{X}, \mathrm{Z}_{1}, \mathrm{Z}_{2}, \mathrm{~W}_{1}, \mathrm{~W}_{2}=$ matrizes de incidência correspondentes; $\mathrm{K}_{\mathrm{A}}, \mathrm{K}_{\mathrm{M}}, \mathrm{K}_{\mathrm{C}}$ e $\mathrm{K}_{\mathrm{Q}}=$ matrizes de variâncias e covariâncias entre os coeficientes de regressão aleatórios para os efeitos genéticos aditivos direto e materno e de ambiente permanente do animal e materno, respectivamente; $\mathrm{A}=$ matriz de parentesco; $\mathrm{I}=$ matriz identidade; $\mathrm{N}_{\mathrm{a}}$ e $\mathrm{N}_{\mathrm{m}}=$ número de animais com dados e número de mães, respectivamente; $\mathrm{R}=$ matriz de variâncias residuais; $\varepsilon=$ vetor de resíduos.

Os componentes de covariância foram estimados por máxima verossimilhança restrita utilizando-se o programa estatístico WOMBAT (Meyer, 2006), ajustando polinômios ortogonais de Legendre em terceira a sexta ordem para modelar os efeitos genéticos aditivos direto e materno, de ambiente permanente de animal e materno. O resíduo foi modelado utilizando-se sete classes de variância, agrupadas da seguinte forma: 0, 1-150, 151-220, 221-260, 261-370, 371-500 e 501-680 dias de idade, respectivamente.

Os modelos de regressão aleatória foram comparados usando-se o logaritmo da função de verossimilhança ( $\log \mathrm{L})$, as formas de máxima verossimilhança restrita dos critérios de informação de Akaike (AIC) e Bayesiano de
Schwarz (BIC), que permitem a comparação de modelos não-aninhados e penalizam modelos mais parametrizados, sendo o BIC é o mais rigoroso, ou seja, o critério que favorece modelos mais parcimoniosos (Wolfinger, 1993). Os critérios de informação podem ser representados como:

$$
\begin{gathered}
\mathrm{AIC}=-2 \log \mathrm{L}+2 \mathrm{p} \\
\mathrm{BIC}=-2 \log \mathrm{L}+\mathrm{p} \log (\mathrm{N}-\mathrm{r}(\mathrm{X})),
\end{gathered}
$$

em que: $\mathrm{p}=$ número de parâmetros do modelo $\mathrm{N}=$ total de observações; e $r$ = posto da matriz X (matriz de incidência para os efeitos fixos). Valores menores de AIC e BIC indicam melhor ajuste do modelo.

Para comparar os resultados obtidos pelos modelos de regressão aleatória, foram realizadas, no mesmo conjunto de dados, análises bicaracterísticas utilizando modelos de dimensão finita para pesos aos 120 (P120), 240 (P240), 365 (P365), 540 (P540) e aos 670 (P670) dias de idade. Foram considerados aleatórios os efeitos genético aditivo direto e residual e, além desses, para P120 e P240 também foram considerados os efeitos genético materno e de ambiente permanente materno. Como efeitos fixos foram utilizados o grupo de contemporâneo (rebanho, ano e estação de nascimento, condição de criação e sexo) e efeitos linear e quadrático da idade do animal à pesagem e idade da vaca ao parto, como covariáveis.

\section{Resultados e Discussão}

No arquivo estudado, há maior concentração de registros nas idades iniciais, ou seja, dos 35 aos 365 dias de idade (Figura 1). As médias dos pesos aumentaram linearmente com a idade, variando de $23 \mathrm{~kg}$ (ao nascimento) a $687 \mathrm{~kg}$ (aos 660 dias de idade). Os desvios-padrão mostraram tendência similar às médias, aumentando com a idade. Os coeficientes de variação tenderam a queda do nascimento até próximo aos 355 dias de idade e, após esta idade, permaneceu constante (Figura 2).

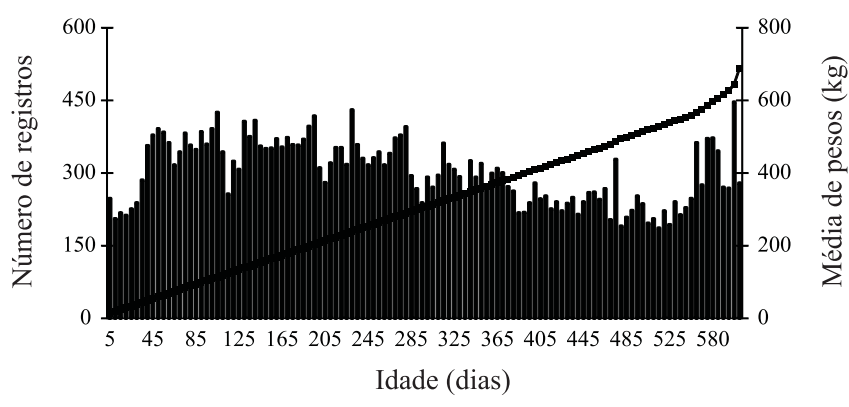

Figura 1 - Número de registros (barras) e média de peso, em kg (linha), de animais da raça Tabapuã em várias idades.

R. Bras. Zootec., v.39, n.5, p.1037-1045, 2010 


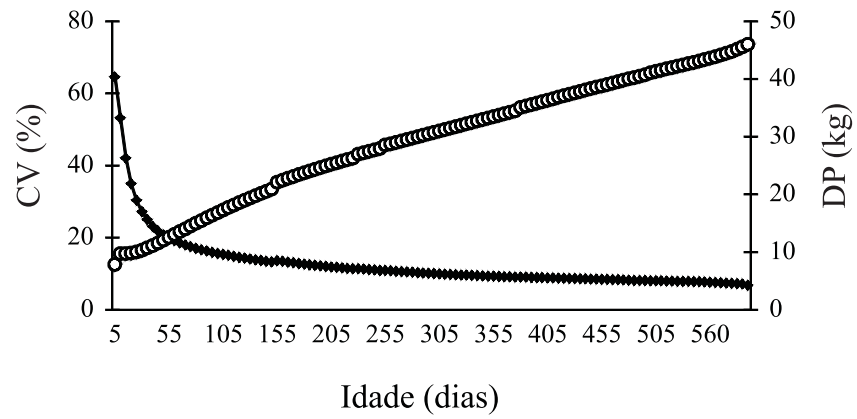

Figura 2 - Coeficiente de variação (\%) e desvio-padrão (kg) para peso, de acordo com a idade de animais da raça Tabapuã.

De acordo com os critérios de informação de AIC, o modelo M5363 para os efeitos genético aditivo direto e materno, de ambiente permanente de animal e materno, respectivamente, com 55 parâmetros foi o melhor. Por outro lado, o BIC indicou o modelo M4363, com um total de 50 parâmetros, suficiente para modelar a variância nos dados (Tabela 2).

Para todos os efeitos aleatórios estudados, o intercepto apresentou sempre a maior estimativa de variância associada (Tabela 3). As correlações entre o intercepto e o coeficiente de regressão linear foram altas e positivas para os efeitos genético aditivo direto $(0,88)$, de ambiente permanente de animal $(0,80)$ e materno $(0,97)$. Para o efeito genético materno, a correlação entre o intercepto e o coeficiente de regressão linear foi negativa $(-0,52)$. Utilizando pesos do nascimento aos 630 dias de idade de animais da raça Nelore, Albuquerque \& Meyer (2001) relataram correlações positivas e altas entre o intercepto e o coeficiente de regressão linear para todos os efeitos aleatórios. De modo semelhante, Dias et al. (2006) também estimaram correlações positivas e altas entre o intercepto e o coeficiente de regressão linear para todos os efeitos aleatórios, utilizando informações de pesos do nascimento aos 550 dias de idade de animais da raça Tabapuã.

De modo geral, as variâncias fenotípicas estimadas no modelo de regressão aleatória foram semelhantes às obtidas em análises bicaracterísticas, as quais apresentaram aumento gradativo no período estudado (Figura 3). Esses resultados diferem dos obtidos por Meyer (2001), Nobre et al. (2003) e Meyer (2005), que descreveram estimativas obtidas com modelos de regressão aleatória superiores em relação ao modelo multicaracterístico, principalmente em idades mais avançadas.

As estimativas de variância genética aditiva direta foram semelhantes às obtidas com a utilização dos modelos bicaracteres (Figura 3). As estimativas de variância genética aditiva direta aumentaram com a idade, passando de $11,74 \mathrm{~kg}^{2}$ (ao nascimento) para 980,21 kg (aos 660 dias de idade). De modo semelhante, aumentos nas estimativas de variância genética aditiva direta durante o crescimento dos animais, porém com magnitudes inferiores, foram relatadas por Albuquerque \& Meyer (2001), Nobre et al. (2003) e Dias et al. (2006), para as raças Nelore e Tabapuã.

Para o efeito de ambiente permanente de animal, observou-se aumento nas estimativas de acordo com a idade e esse aumento foi mais acentuado na fase final de crescimento. O aumento das estimativas de variância de ambiente permanente de animal para pesos a partir da desmama também foi relatado por Albuquerque \& Meyer (2001) e Dias et al. (2006).

As variâncias genética materna e de ambiente permanente materno estimadas utilizando análises bicaracterísticas foram semelhantes às obtidas com o modelo de regressão aleatória. Com a utilização desse último modelo, as estimativas de variância genética materna aumentaram do nascimento até próximo aos 200 dias de idade, com leve tendência de queda nas idades futuras, voltando a aumentar no final do período estudado (Figura 3). Por outro lado, as variâncias estimadas para o efeito de ambiente permanente materno aumentaram do nascimento aos 660 dias de idade. Resultados semelhantes foram descritos por Albuquerque \& Meyer (2001), Nobre et al. (2003) e Dias et al. (2006).

As herdabilidades para o efeito aditivo direto estimadas com análises bicaracterísticas e por regressão aleatória foram semelhantes, em tendência, durante todo o período estudado, mas as obtidas por regressão aleatória foram, em geral, mais altas (Figura 4). As herdabilidades

Tabela 2 - Resumo dos modelos estudados

\begin{tabular}{|c|c|c|c|c|c|c|c|c|}
\hline \multirow[t]{2}{*}{ Modelo } & \multicolumn{8}{|c|}{ Critério estatístico ${ }^{1}$} \\
\hline & $\mathrm{k}_{\mathrm{a}}$ & $\mathrm{k}_{\mathrm{m}}$ & $\mathrm{k}_{\mathrm{c}}$ & $\mathrm{k}_{\mathrm{q}}$ & $\mathrm{np}$ & $\log$ & AIC & BIC \\
\hline M4252 & 4 & 2 & 5 & $2^{4}$ & 38 & $-97613,610$ & 195309,220 & 195653,940 \\
\hline M4352 & 4 & 3 & 5 & 2 & 41 & $-95595,697$ & 191273,394 & 191618,114 \\
\hline M4353 & 4 & 3 & 5 & 3 & 44 & $-94014,054$ & 188116,108 & 188486,051 \\
\hline M4363 & 4 & 3 & 6 & 3 & 50 & $-91344,452$ & 182788,904 & 183209,294 \\
\hline M5363 & 5 & 3 & 6 & 3 & 55 & $-91324,183$ & 182758,366 & 183220,795 \\
\hline
\end{tabular}

${ }^{1}$ Ordem dos polinômios: efeito genético direto $\left(\mathrm{k}_{\mathrm{a}}\right)$, efeito genético materno $\left(\mathrm{k}_{\mathrm{m}}\right)$, efeito de ambiente permanente de animal $\left(\mathrm{k}_{\mathrm{c}}\right)$, efeito de ambiente permanente materno $\left(\mathrm{k}_{\mathrm{q}}\right)$; número de parâmetros estimados (np); logaritmo da função de verossimilhança (log); critério de informação de Akaike (AIC); critério de informação Bayesiano de Schwarz (BIC). 
diretas obtidas em análises bicaracterísticas foram de 0,28 (P120); 0,31 (P240); 0,40 (P365); 0,44 (P540) e 0,46 P(670)e as herdabilidades maternas foram de 0,05 e 0,02 para $\mathrm{P} 120$ e P365, respectivamente.

As estimativas de herdabilidade direta obtidas utilizando modelo de regressão aleatória apresentaram pequena tendência de queda do nascimento $(0,15)$ até os 20 dias $(0,11)$ e, após esta idade,tenderam a aumento, atingindo 0,45 aos 660 dias de idade (Figura 4). Meyer (2001) descreveu diminuição das estimativas de herdabilidade após o nascimento com posterior aumento a partir da desmama para pesos de gado de corte na Austrália. Albuquerque \& El Faro (2008) também apresentaram tendência semelhante, porém com magnitudes inferiores, utilizando pesos de animais da raça Nelore.

As herdabilidades diretas estimadas neste estudo são próximas aos valores descritos na literatura para zebuínos, os quais são, em média de 0,30 (peso à desmama), 0,39 (peso aos 365 dias de idade) e 0,46 (peso ao sobreano) (Cabrera et al., 2001; Ferraz Filho et al., 2002; Talhari et al., 2003; Forni \& Albuquerque, 2005; Pereira et al., 2005). Por outro lado, existem autores que, utilizando modelos de regressão aleatória, relataram estimativas de herdabilidade direta com tendência de redução entre o nascimento e a desmama e posterior aumento até o sobreano (Meyer, 2001; Albuquerque \& El Faro, 2008; Albuquerque \& Meyer, 2001; Nobre at al., 2003; Dias et al., 2006).

Em geral, as herdabilidades diretas estimadas e a variância genética aditiva direta sugerem haver variabilidade genética suficiente para a obtenção de ganho genético mais acentuado se a seleção individual for realizada considerando pesos após à desmama, uma vez que, neste período será possível melhor identificação dos animais geneticamente superiores. Ressalta-se que, quanto mais tardia a seleção para pesos, maior a chance de aumento do peso adulto das matrizes, uma vez que a correlação entre pesos a partir do sobreano com o peso adulto é alta e que aumento no peso adulto de matrizes nem sempre é desejável (Boligon et al., 2008).

As herdabilidades maternas apresentaram baixas magnitudes e foram semelhantes às descritas por Mercadante et al. (2000); Cabrera et al. (2001) e Bertazzo et al. (2004). Por outro lado, utilizando modelos de regressão aleatória, Albuquerque \& Meyer (2001), Dias et al. (2006) e Nobre et al. (2003) estimaram maiores herdabilidades maternas para pesos do nascimento ao sobreano. Segundo Meyer (1992), a divisão dos efeitos maternos em genético e de ambiente permanente não é simples, principalmente com dados não-experimentais e existe a possibilidade de considerar apenas um dos efeitos maternos nos procedimentos de análises.

As estimativas obtidas neste estudo indicam influência materna crescente até os 150 dias de idade. Portanto, embora de baixa magnitude, maior resposta a seleção para habilidade materna em rebanhos da raça Tabapuã pode ser esperada com à seleção realizada com base em pesos obtidos próximo à desmama. Resultados semelhantes foram relatados por Albuquerque \& Meyer (2001) para animais da raça Nelore e por Dias et al. (2006) para animais da raça Tabapuã. É importante ressaltar que a seleção para habilidade materna deve resultar em fêmeas com maior potencial genético para produção de leite, pois esta característica é o principal componente do efeito materno.

As estimativas de variância de ambiente permanente de animal como proporção da variância fenotípica total, obtidas por regressão aleatória, apresentaram tendência de

Tabela 3 - Estimativas de variâncias (diagonal), covariâncias (abaixo da diagonal) e correlações (acima da diagonal) entre os coeficientes de regressão aleatória e seus autovalores $(\lambda)$ para o modelo M4363

\begin{tabular}{|c|c|c|c|c|c|c|c|c|}
\hline Item & & 0 & 1 & 2 & 3 & 4 & 5 & $\lambda$ \\
\hline \multirow[t]{4}{*}{ Genético aditivo } & 0 & 602,71 & 0,88 & $-0,28$ & $-0,09$ & & & 748,91 \\
\hline & 1 & 288,12 & 177,39 & 0,05 & $-0,45$ & & & 41,68 \\
\hline & 2 & $-23,89$ & 2,26 & 12,11 & $-0,15$ & & & 7,66 \\
\hline & 3 & $-5,68$ & $-15,77$ & $-1,44$ & 6,84 & & & 0,80 \\
\hline \multirow[t]{3}{*}{ Genético materno } & 0 & 0,37 & $-0,52$ & $-0,69$ & & & & 20,36 \\
\hline & 1 & $-1,21$ & 14,71 & 0,97 & & & & 0,45 \\
\hline & 2 & $-1,01$ & 8,96 & 5,73 & & & & 0,00 \\
\hline \multirow{5}{*}{ Ambiente permanente do animal } & 1 & 143,95 & 81,15 & $-0,21$ & $-0,22$ & 0,24 & 0,21 & 35,13 \\
\hline & 2 & $-55,42$ & $-8,42$ & 19,48 & $-0,08$ & $-0,23$ & 0,12 & 10,46 \\
\hline & 3 & 10,91 & $-5,58$ & $-1,03$ & 7,95 & $-0,03$ & $-0,75$ & 3,72 \\
\hline & 4 & 4,54 & 3,88 & $-1,85$ & $-0,15$ & 3,28 & 0,57 & 0,11 \\
\hline & 5 & $-3,82$ & 1,71 & 0,48 & $-1,87$ & 0,91 & 0,79 & 0,00 \\
\hline \multirow[t]{2}{*}{ Ambiente permanente materno } & 0 & 290,08 & 0,97 & $-0,92$ & & & & 343,44 \\
\hline & 1 & 120,13 & 53,31 & $-0,79$ & & & & 3,69 \\
\hline
\end{tabular}


crescimento do nascimento $(0,01)$ até os 160 dias de idade $(0,29)$ com suave diminuição até os 440 dias de idade $(0,23)$ e, a partir dessa idade, permaneceram constantes (0,24). Por outro lado, as estimativas de variância de ambiente permanente materno, como proporção da variância fenotípica total aumentaram suavemente com a idade, variando de 0,02 (ao nascimento) a 0,15 (aos 660 dias de idade). Esses resultados assemelham-se aos descritos por Meyer (2001) para animais da raça Polled Hereford.
As correlações genéticas estimadas entre o peso ao nascer e os pesos até os 365 dias de idade foram positivas, de moderadas a altas magnitudes, com tendência de diminuição após esse período (Tabela 4). Portanto, a seleção com base nos pesos obtidos em idades jovens pode levar a aumento do peso ao nascimento e, como conseqüência, elevar a incidência de partos distócicos. Resultados semelhantes foram obtidos por Boligon et al. (2008), os quais estimaram correlações genéticas de 0,81 (peso ao
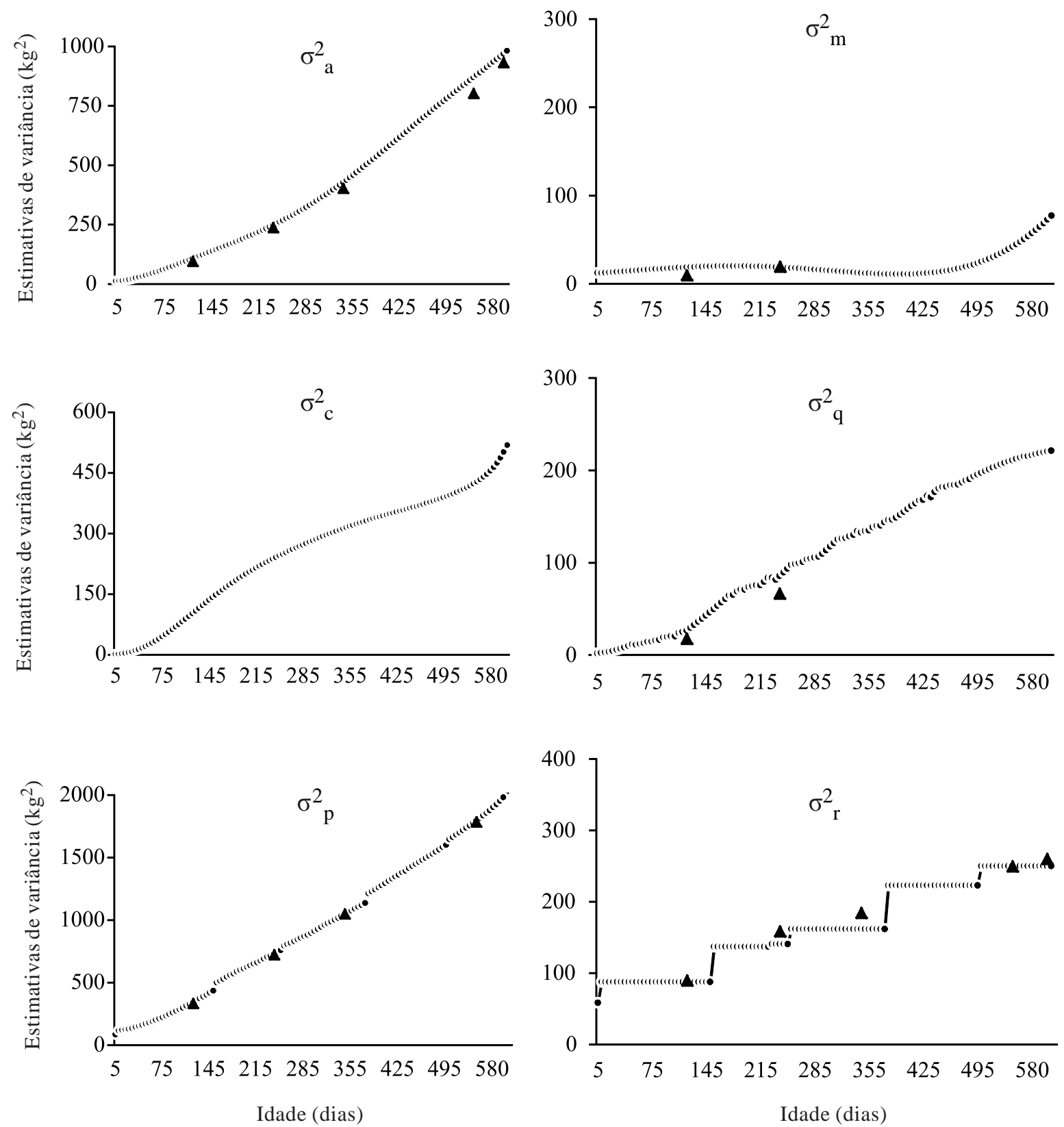

Figura 3 - Estimativas de componentes de variância genética aditiva direta (acima à esquerda), genética materna (acima à direita), de ambiente permanente de animal (centro à esquerda), de ambiente permanente materno (centro à direita), fenotípica (abaixo à direita) e residual (abaixo à esquerda) utilizando modelo de regressão aleatória (0) e bicaracterística (ム).

R. Bras. Zootec., v.39, n.5, p.1037-1045, 2010 
nascer $\times$ peso à desmama) e $0,74($ peso ao nascer $\times$ peso ao sobreano).

As correlações genéticas estimadas entre os pesos da desmama aos 660 dias de idade foram altas e positivas e indicam que a maior parte dos genes responsáveis por maiores pesos nesse período são os mesmos. Tais resultados estão de acordo com os descritos por Ferraz Filho et al. (2002), Santos et al. (2005) e Boligon et al. (2008), que variaram de 0,61 a 0,83 entre o peso à desmama e ao sobreano. Em análise multivariada, Meyer et al. (1993) estimaram correlação genética de 0,92 entre o peso à desmama e o peso ajustado aos 600 dias de idade.

As correlações de ambiente permanente de animal apresentaram magnitudes altas entre as idades padrão, ou seja, 0,92 (entre P240 e P365), 0,80 (entre P240 e P550) e 0,90 (entre P365 e P550). Estimativas inferiores foram relatadas por Albuquerque \& Meyer (2001) e Dias et al. (2006) para animais da raça Nelore e Tabapuã.

As estimativas de correlação genética materna entre o peso ao nascer e as idades padrão diminuíram com o aumento da distância entre as idades: 0,$81 ; 0,39$ e 0,15 entre o peso ao nascimento e aos 240, 365 e 550 dias de idade, respectivamente. Para pesos obtidos da desmama aos 600 dias de idade, as correlações chegaram a 0,66. Eler et al. (1995) obtiveram estimativas baixas de correlação genética materna entre os pesos ao nascer e à desmama $(0,21)$ e entre peso à desmama e ao sobreano $(0,18)$ e correlação alta entre o peso à desmama e ao ano $(0,84)$ para animais da raça Nelore. Albuquerque \& Meyer (2001) relataram baixos valores de correlação genética materna entre o peso ao nascimento e aos 240,360 e 550 dias de idade $(0,14$; 0,05 e 0,05 , respectivamente) e correlações próximas à unidade entre as demais idades. Nobre et al. (2003) estimaram baixas correlações genéticas maternas entre o peso ao nascer e os pesos próximos aos 60 dias de idade $(0,20)$.

As estimativas de correlaçãos de ambiente permanente materno diminuíram à medida que aumentou a distancia entre as idades, e apresentaram valores de 0,$53 ; 0,45 ; 0,31$ e 0,27 entre o peso ao nascer e os pesos aos 240,365, 550 e 660 dias de idade, respectivamente. Após a desmama, as estimativas apresentaram valores de alta magnitude, próxima à unidade, ou seja, 0,99 (P240× P365); 0,97 (P240× P550) e $0,96(\mathrm{P} 365 \times \mathrm{P} 550)$.

As estimativas de correlação fenotípica diminuíram à medida que os pesos se distanciavam, porém, as correlações entre o peso ao nascimento e os pesos posteriores foram de baixa magnitude (Tabela 4). Já para pesos a partir da desmama, os valores foram superiores a 0,66. Essa expressiva relação linear entre os pesos após o nascimento indica que a seleção para maior peso nas primeiras idades tende a aumentar o pesonas idades mais avançadas e viceversa. Resultados semelhantes foram verificados por Dias et al. (2006), que estimaram correlações fenotípicas semelhantes entre o peso ao nascer e o peso à desmama $(0,27)$ e entre os pesos da desmama aos 550 dias de idade $(0,13$ a 0,26$)$. Para as idades mais avançadas, no entanto, as correlações fenotípicas foram maiores, chegando a 0,94 nos períodos entre 240 a 365 dias de idade e 0,61 a 0,74 e entre 240 e 550 dias de idade.

Segundo Dias et al. (2006), as estimativas de parâmetros genéticos podem ser influenciadas pelo modelo de regressão adotado. A partição dos efeitos aleatórios separando os efeitos genéticos e de ambiente em direto e materno é essencial para modelar as mudanças que ocorrem de maneira adequada. A modelagem do resíduo e a ordem do ajuste dos polinômios podem interferir na estimação dos componentes de variância.

A literatura apresenta vários trabalhos que indicam que considerar estruturas heterogêneas de variância residual é mais adequado para ajustar pesos de bovinos ao longo do crescimento. Assumir homogeneidade de variância significa que o ambiente temporário afeta igualmente todas as idades, o que nem sempre ocorre. Além disso, grande parte da variação residual pode contaminar a variância de ambiente permanente de animal (Huisman et al., 2002).

Tabela 4 - Estimativas de correlações genética (acima da diagonal) e fenotípica (abaixo da diagonal) para pesos em diferentes idades em animais da raça Tabapuã

\begin{tabular}{lcccc}
\hline Característica & Peso ao nascer & Peso aos 240 dias & Peso aos 365 dias & Peso aos 540 dias \\
\hline Peso ao nascer & - & 0,79 & 0,77 & 0,64 \\
Peso aos 240 dias & 0,25 & - & 0,94 & 0,85 \\
Peso aos 365 dias & 0,21 & 0,79 & - & 0,54 \\
Peso aos 540 dias & 0,27 & 0,69 & 0,81 & 0,84 \\
Peso aos 660 dias & 0,30 & 0,67 & 0,77 & 0,93 \\
\hline
\end{tabular}



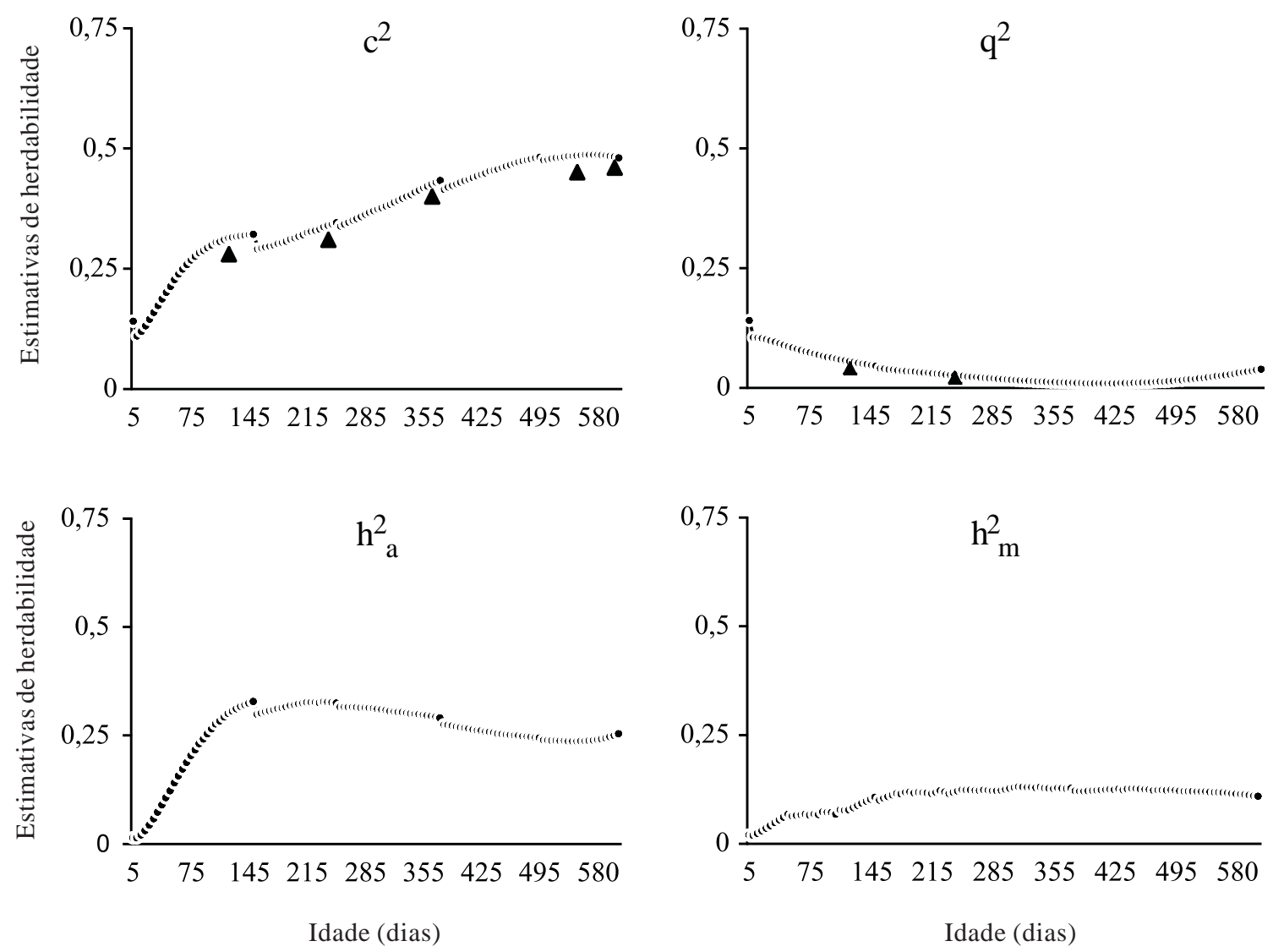

Figura 4 - Estimativas de herdabilidade direta $\left(\mathrm{h}^{2}{ }_{\mathrm{a}}\right)$ e materna $\left(\mathrm{h}_{\mathrm{m}}^{2}\right)$, variância de ambiente permanente de animal $\left(\mathrm{c}^{2}\right)$ e materna $\left(\mathrm{q}^{2}\right)$ como proporção da variância fenotípica total de pesos do nascimento aos 660 dias de idade utilizando modelo de regressão aleatória (0) e bicaracterística $(\mathbf{\Delta})$.

\section{Conclusões}

As estimativas de covariância e herdabilidades obtidas utilizando modelo bicaracter e de regressão aleatória são semelhantes. Os modelos de regressão aleatória são adequados para descrever as mudanças nas variâncias dos pesos do nascimento aos 660 dias de idade de animais da raça Tabapuã criados no estado da Bahia. A seleção para maiores pesos em qualquer idade promove aumentos nos pesos do nascimento e aos 660 dias de idade. Entretanto, a seleção com base nos pesos pré-desmama promove resposta, apesar de pequena, na habilidade materna.

\section{Referências}

ASSOCIAÇÃO BRASILEIRA DOS CRIADORES DE ZEBU - ABCZ. As raças Zebuínas. [2008]. Disponível em: <http:// www.abcz.org.br/site/tecnica/racas/tab.php>. Acesso em: 18/3/ 2008.

ALBUQUERQUE, L.G.; EL FARO, L. Comparação entre os valores genéticos para características de crescimento de bovinos da raça Nelore preditos com modelos de dimensão finita e infinita Revista Brasileira de Zootecnia, v.37, n.2, p.238-246, 2008 .

ALBUQUERQUE, L.G.; MEYER, K. Estimates of covariance functions for growth from birth to 630 days of age in Nelore cattle. Journal of Animal Science, v.79, p.2776-2789, 2001 .

BERTAZZO, R.P.; FREITAS, R.T.F.; GONÇALVES, T.M. et al Parâmetros genéticos de longevidade e produtividade de fêmeas da raça Nelore. Revista Brasileira de Zootecnia, v.33, n.5, p.1118-1127, 2004.

BOLIGON, A.A.; ALBUQUERQUE, L.G.; RORATO, P. N. et al Associações genéticas entre pesos e características reprodutivas em rebanhos da raça Nelore. Revista Brasileira de Zootecnia v.37, n.4, p.596-601, 2008.

CABRERA, M.E.; GARNERO, A.V.; LÔBO, R.B. et al. Efecto de la incorporación de la covarianza genética directa-materna em el análisis de características de crecimiento em la raza Nelore. Livestock Research for Rural Development, v.13, n.3 2001

DIAS, L.T.; ALBUQUERQUE, L.G.; TONHATI, H. Estimação de parâmetros genéticos para peso em diferentes idades para animais da raça Tabapuã. Revista Brasileira de Zootecnia, v.35, n.5 p.1915-1925, 2006

ELER, J.P.; VAN VLECK, L.D.; FERRAZ, J.B.S.; LÔBO, R.B Estimation of variances due to dierct and maternal effects for growth traits in Nelore cattle. Journal of Animal Science, v.73, p.3253-3258, 1995.

R. Bras. Zootec., v.39, n.5, p.1037-1045, 2010 
FERRAZ FILHO, P.B.; RAMOS, A.A.; SILVA, L.O.C. et al. Herdabilidade e correlações genéticas, fenotípicas e ambientais para pesos em diferentes idades de bovinos da raça Tabapuã. Archives of Veterinary Science, v.7, n.1, p.65-69, 2002.

FORNI, S.; ALBUQUERQUE, L. G. Estimates of genetics correlations between days to calving and reproductive and weight traits in Nelore cattle. Journal of Animal Science, v.83, p.1511-1515, 2005

HUISMAN, A.E.; VEERKAMP, R.F.; ARENDONK, J.A.M. Genetics parameters for various random regression models to describe the weight data of pigs. Journal of Animal Science, v.80, p.575-582, 2002.

MERCADANTE, M.E.Z.; LÔBO, R.B.; OLIVEIRA, H.N. Estimativas de (co)variância entre características de reprodução e de crescimento em fêmeas de um rebanho Nelore. Revista Brasileira de Zootecnia, v.29, n.4, p.997-1004, 2000.

MEYER, K. Bias and sampling covariances of estimates of variances components due to maternal effects. Genetics Selection Evolution, v.24, p.487-509, 1992.

MEYER, K.; CARRICK, M.J.; DONNELLY, B.J.P. Genetic parameters for growth traits of Australian beef cattle from a multibreed selection experiment. Journal of Animal Science, v.71, p.2614-2622, 1993.

MEYER, K.; HILL, W.G. Estimation of genetic and phenotypic covariance functions for longitudinal 'repeated' records by restrict maximum likelihood. Livestock Production Science, v.47, n.3, p.85-200, 1997.

MEYER, K. Estimates of direct and maternal covariance functions for growth of Australian beef calves from birth to weaning. Genetics Selection Evolution, v.33, p.1-28, 2001
MEYER, K. Estimates of genetic covariance functions for growth of Angus cattle. Journal of Animal Breeding and Genetic, v.122, p.73-85, 2005.

MEYER, K. "WOMBAT" - Digging deep for quantitative genetic analyses by restricted maximum likelihood. In: WORLD CONGRESS OF GENETICS, 8., 2006, Belo Horizonte. Proceedings... Belo Horizonte, 2006 (CD-ROM).

NOBRE, P.R.C.; MISZTAL, I.; TSURUTA, S. et al. Analyses of grwth curves of Nellore cattle by multiple-trait and random regression models. Journal of Animal Science, v.81, p.918-926, 2003.

PEREIRA, J.C.C.; RIBEIRO, S.H.A.; SILVA, M.A. et al. Análise genética de características ponderais e reprodutivas de fêmeas bovinas Tabapuã. Arquivo Brasileiro de Medicina Veterinária e Zootecnia, v.57, p.231-236, 2005.

SAKAGUTI, E.S.; SILVA, M.A.; QUAAS, R.L. et al. Avaliação do crescimento de bovinos jovens da raça Tabapuã, por meio de análises de funções de covariâncias. Revista Brasileira de Zootecnia, v.32, n.4, p.864-874, 2003.

SANTOS, P.F.; MALHADO, C.H.M.M.; CARNEIRO, P.L.S. et al Correlação genética, fenotípica e ambiental em características de crescimento de bovinos da raça Nelore variedade mocha Archives of Veterinary Science, v.10, n.2, p.55-60, 2005.

TALHARI, F.M.; ALENCAR, M.M.; MASCIOLI, A.S. et al Correlações genéticas entre características produtivas de fêmeas em um rebanho da raça Canchim. Revista Brasileira de Zootecnia, v.32, n.4, p.880-886, 2003.

WOLFINGER, R. Covariance structure selection in general mixed models. Communications in Statistics, v.22, n.4 p.1079-1106, 1993. 